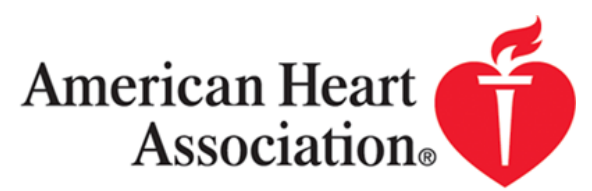

Learn and Live

\title{
Albuminuria, a Therapeutic Target for Cardiovascular Protection in Type 2 Diabetic Patients With Nephropathy
}

Dick de Zeeuw, Giuseppe Remuzzi, Hans-Henrik Parving, William F. Keane, Zhongxin Zhang, Shahnaz Shahinfar, Steve Snapinn, Mark E. Cooper, William E. Mitch and Barry M. Brenner

Circulation 2004;110;921-927; originally published online Aug 9, 2004;

DOI: 10.1161/01.CIR.0000139860.33974.28

Circulation is published by the American Heart Association. 7272 Greenville Avenue, Dallas, TX 72514

Copyright $@ 2004$ American Heart Association. All rights reserved. Print ISSN: 0009-7322. Online ISSN: $1524-4539$

The online version of this article, along with updated information and services, is located on the World Wide Web at:

http://circ.ahajournals.org/cgi/content/full/110/8/921

Subscriptions: Information about subscribing to Circulation is online at http://circ.ahajournals.org/subscriptions/

Permissions: Permissions \& Rights Desk, Lippincott Williams \& Wilkins, a division of Wolters Kluwer Health, 351 West Camden Street, Baltimore, MD 21202-2436. Phone: 410-528-4050. Fax: 410-528-8550. E-mail:

journalpermissions@lww.com

Reprints: Information about reprints can be found online at http://www.lww.com/reprints 


\title{
Albuminuria, a Therapeutic Target for Cardiovascular Protection in Type 2 Diabetic Patients With Nephropathy
}

\author{
Dick de Zeeuw, MD, PhD; Giuseppe Remuzzi, MD; Hans-Henrik Parving, MD; \\ William F. Keane, MD; Zhongxin Zhang, PhD; Shahnaz Shahinfar, MD; Steve Snapinn, PhD; \\ Mark E. Cooper, MD, PhD; William E. Mitch, MD; Barry M. Brenner, MD
}

Background - Albuminuria is an established risk marker for both cardiovascular and renal outcomes. Albuminuria can be reduced with drugs that block the renin-angiotensin system (RAS). We questioned whether the short-term drug-induced change in albuminuria would predict the long-term cardioprotective efficacy of RAS intervention.

Methods and Results - We analyzed data from Reduction in Endpoints in Non-insulin dependent diabetes mellitus with the Angiotensin II Antagonist Losartan (RENAAL), a double-blind, randomized trial in 1513 type 2 diabetic patients with nephropathy, focusing on the relationship between the prespecified cardiovascular end point (composite) or hospitalization for heart failure and baseline or reduction in albuminuria. Patients with high baseline albuminuria $(\geq 3$ $\mathrm{g} / \mathrm{g}$ creatinine) had a 1.92-fold ( $95 \% \mathrm{CI}, 1.54$ to 2.38 ) higher risk for the cardiovascular end point and a 2.70 -fold (95\% CI, 1.94 to 3.75) higher risk for heart failure compared with patients with low albuminuria $(<1.5 \mathrm{~g} / \mathrm{g})$. Among all available baseline risk markers, albuminuria was the strongest predictor of cardiovascular outcome. The association between albuminuria and cardiovascular outcome was driven by those patients who also had a renal event. Modeling of the initial 6-month change in risk parameters showed that albuminuria reduction was the only predictor for cardiovascular outcome: $18 \%$ reduction in cardiovascular risk for every 50\% reduction in albuminuria and a $27 \%$ reduction in heart failure risk for every $50 \%$ reduction in albuminuria.

Conclusions-Albuminuria is an important factor predicting cardiovascular risk in patients with type 2 diabetic nephropathy. Reducing albuminuria in the first 6 months appears to afford cardiovascular protection in these patients. (Circulation. 2004;110:921-927.)

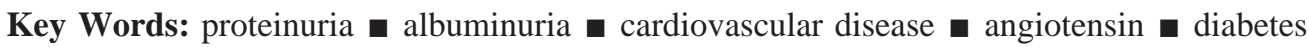

$I_{n}^{t}$ is estimated that type 2 diabetes affects more than 150 million people worldwide, and the prevalence is projected to double in the next 25 years. ${ }^{1}$ Nephropathy, characterized by albuminuria, hypertension, and a progressive decline in glomerular filtration rate, develops in $10 \%$ to $40 \%$ of diabetic patients. ${ }^{2}$ An associated progressive loss of cardiovascular function leads to a predominantly cardiovascular mortality in this population. ${ }^{3}$ Treatment of type 2 diabetes has focused on attenuating hyperglycemia, hypertension, and dyslipidemia. ${ }^{4-6}$ Indeed, both blood pressure and hyperglycemia have not only proved to be important risk markers, but their control also serves as an indicator of the effectiveness of cardiovascular-protective therapy. ${ }^{7-9}$

Albuminuria in type 2 diabetic patients has been identified as another risk marker for both cardiovascular ${ }^{10}$ and renal outcome. ${ }^{11}$ Several antiproteinuric strategies are available, including interruption of the renin-angiotensin system (RAS) via either ACE inhibitor (ACEI) or angiotensin II antagonists (AIIA). Reduction in albuminuria using such drug strategies appears to be related to renal protection independent of blood pressure effects. ${ }^{12,13}$ This phenomenon was recently also documented in type 2 diabetes. ${ }^{14}$ Whether lowering albuminuria would reduce the subsequent risk for cardiovascular events has not been documented.

We therefore sought to confirm that albuminuria is an independent cardiac risk marker in type 2 diabetes with nephropathy. We investigated whether albuminuria reduction is associated with a reduction in cardiovascular events and whether the degree of the short-term therapy-induced albuminuria reduction would be an indicator of the subsequent long-term cardiovascular protection. Finally, we questioned whether albuminuria-driven cardiovascular risk is associated with the renal risk in the same patient. If these issues are answered positively, it is predicted that albuminuria may

Received July 22, 2003; de novo received November 17, 2003; revision received April 20, 2004; accepted April 22, 2004.

From the Department of Clinical Pharmacology, Groningen University Medical Center, the Netherlands (D.d.Z.); Mario Negri Institute for Pharmacological Research, Bergamo, Italy (G.R.); Steno Diabetes Center Gentofte and Faculty of Health Science, Aarhus University, Denmark (H.-H.P.); Merck Research Laboratories, Merck \& Co, Inc, Whitehouse Station, NJ (W.F.K., Z.Z., S. Shahinfar, S. Snapinn); Baker Medical Research Institute, Melbourne, Australia (M.E.C.); Department of Medicine, University of Texas, Medical Branch, Galveston (W.E.M.); and Department of Medicine, Renal Division, Brigham and Women's Hospital and Harvard School of Medicine, Boston, Mass (B.M.B.).

Correspondence to Prof Dr D. de Zeeuw, Department of Clinical Pharmacology, Groningen University Medical Center, Ant Deusinglaan 1, 9713 AV Groningen, Netherlands. E-mail d.de.zeeuw@med.rug.nl

(C) 2004 American Heart Association, Inc.

Circulation is available at http://www.circulationaha.org 
TABLE 1. Baseline Characteristics

\begin{tabular}{|c|c|c|c|c|c|c|c|c|c|}
\hline & \multicolumn{3}{|c|}{ Losartan $(n=751)$} & \multicolumn{3}{|c|}{ Placebo $(n=762)$} & \multicolumn{3}{|c|}{ Total $(n=1513)$} \\
\hline & $\mathrm{n}$ & Mean & SD & $\mathrm{n}$ & Mean & SD & $\mathrm{n}$ & Mean & SD \\
\hline Sex, female/male & $289 / 462$ & & & $268 / 494$ & & & $557 / 956$ & & \\
\hline Smoking, ${ }^{*}$ yes/no & $145 / 604$ & & & $128 / 632$ & & & $273 / 1236$ & & \\
\hline CV disease history & $328 / 751$ & & & $327 / 762$ & & & $655 / 1513$ & & \\
\hline HF disease history & $41 / 751$ & & & $41 / 762$ & & & $82 / 1513$ & & \\
\hline Age, y & 751 & 60.0 & 7.4 & 762 & 60.3 & 7.5 & 1513 & 60.2 & 7.4 \\
\hline Weight, kg & 751 & 82.6 & 20.6 & 762 & 81.7 & 20.9 & 1513 & 82.2 & 20.7 \\
\hline SiSBP, mm Hg & 751 & 151.8 & 18.7 & 762 & 153.2 & 19.9 & 1513 & 152.5 & 19.3 \\
\hline SiDBP, mm Hg & 751 & 82.4 & 10.3 & 762 & 82.4 & 10.6 & 1513 & 82.4 & 10.4 \\
\hline MAP, mm Hg & 751 & 105.5 & 10.9 & 762 & 106.0 & 11.6 & 1513 & 105.8 & 11.3 \\
\hline Pulse pressure, $\mathrm{mm} \mathrm{Hg}$ & 751 & 69.4 & 17.4 & 762 & 70.8 & 18.1 & 1513 & 70.1 & 17.8 \\
\hline Cholesterol, mg/dL & 743 & 227.4 & 55.6 & 755 & 228.7 & 55.4 & 1498 & 228.1 & 55.5 \\
\hline $\mathrm{GFR}, \mathrm{mL} \cdot \mathrm{min}^{-1} \cdot 1.73 \mathrm{~m}^{-2}$ & 751 & 39.7 & 12.0 & 762 & 40.0 & 12.7 & 1513 & 39.8 & 12.3 \\
\hline $\mathrm{HbA}_{1 \mathrm{C}}, \%$ & 742 & 8.5 & 1.7 & 754 & 8.4 & 1.6 & 1496 & 8.5 & 1.6 \\
\hline Hemoglobin, g/dL & 732 & 12.5 & 1.8 & 736 & 12.5 & 1.8 & 1468 & 12.5 & 1.8 \\
\hline $\mathrm{S}$ creatinine, $\mathrm{mg} / \mathrm{dL}$ & 751 & 1.9 & 0.5 & 762 & 1.9 & 0.5 & 1513 & 1.9 & 0.5 \\
\hline Albuminuria, $\mathrm{g} / \mathrm{g}$ & 751 & 1.87 & 1.83 & 762 & 1.74 & 1.54 & 1513 & 1.81 & 1.69 \\
\hline Geometric mean & & 1.17 & $\ldots$ & & 1.15 & $\cdots$ & & 1.16 & $\ldots$ \\
\hline Proteinuria, g/d $\dagger$ & 350 & 3.48 & 3.49 & 360 & 3.49 & 3.59 & 710 & 3.49 & 3.54 \\
\hline Geometric mean & & 2.23 & $\ldots$ & & 2.22 & $\ldots$ & & 2.22 & $\ldots$ \\
\hline
\end{tabular}

become the target of a cardiovascular-protection treatment strategy beyond or in addition to blood pressure reduction. To this end, we performed a post hoc analysis of the Reduction in Endpoints in Non-insulin dependent diabetes mellitus with the Angiotensin II Antagonist Losartan (RENAAL) database. The overall RENAAL results have shown a beneficial effect of losartan on the "first hospitalization for heart failure" component of the secondary, cardiovascular end point, whereas the outcome of the cardiovascular composite end point was similar in the 2 treatment arms. ${ }^{15}$

\section{Methods}

\section{Patients and Study Design}

RENAAL is a multinational, double-blind, randomized trial comparing losartan with placebo, each in addition to conventional antihypertensive therapy, excluding ACEIs and other AIIAs. The study was performed in 250 centers in 28 countries and involved 1513 patients. The study design, the inclusion/exclusion criteria, and the treatment protocol have been reported previously. ${ }^{16}$ In short, participants had to have had type 2 diabetes and nephropathy, evidenced by a urinary albumin:creatinine ratio $>0.3 \mathrm{~g} / \mathrm{g}$ in a first morning void or a 24-hour urine protein $>0.5 \mathrm{~g}$, and serum creatinine $>1.5 \mathrm{mg} / \mathrm{dL}(1.3$ $\mathrm{mg} / \mathrm{dL}$ in women or in men $<60 \mathrm{~kg}$ ) to $3.0 \mathrm{mg} / \mathrm{dL}$. We excluded patients who had sustained a myocardial infarction or had undergone coronary artery bypass graft surgery within the previous month, had a cerebrovascular accident or had undergone percutaneous transluminal coronary angioplasty within the previous 6 months, had a transient ischemic attack within the previous year, or had any history of heart failure before enrollment. All patients signed informed consent before enrollment, and the local Institutional Review Board of each participating center approved the study. Patients were followed up for an average of 3.4 years.

\section{Data Analysis}

Albuminuria was assessed using the albumin (g/L)-to-creatinine $(\mathrm{g} / \mathrm{L})$ ratio. For the initial albuminuria response, the month- 6 change was chosen, expressed as $100 \% \times(1-$ ratio of albuminuria month- 6 over baseline).

The cardiovascular end point was defined as the composite of myocardial infarction, stroke, first hospitalization for heart failure or unstable angina, coronary or peripheral revascularization, or cardiovascular death. We analyzed the post hoc, non-heart failure cardiovascular end point, which consisted of all components of the cardiovascular end point, with the exception of hospitalization for heart failure. The renal end point is defined as the composite of the time to first doubling of serum creatinine, end-stage renal disease (ESRD), or death. For patients who had multiple end points of different types, the patients were counted once for the first event in each relevant analysis.

\section{Statistical Analysis}

All 1513 participants in the RENAAL study were included in the analysis from randomization through study termination. Independent baseline and month- 6 predictors of the respective cardiovascular and heart failure end points were identified by the multivariate Cox model. For the baseline analysis, the Cox model included cardiovascular disease history (yes/no) and heart failure disease history (yes/no), age (y/10), sex, race, weight, smoking, sitting systolic blood pressure (SiSBP), sitting diastolic BP (SiDBP), mean arterial pressure, pulse pressure, total cholesterol, estimated glomerular filtration rate, hemoglobin, $\mathrm{HBA}_{1 \mathrm{C}}$, albuminuria, and treatment (losartan/placebo). For the baseline and month- 6 change multivariate analysis, the multivariate Cox model included the baseline risk factors described above and month- 6 changes and log-changes from baseline for the following risk factors: albuminuria, SiDBP, SiSBP, estimated glomerular filtration rate, weight, and $\mathrm{HbA}_{1 \mathrm{C}}$. For all multivariate analyses, a backward selection method was used with the significance 


\begin{tabular}{|c|c|c|c|c|c|}
\hline End Point & Multivariate Risk Factors & $\mathrm{HR}$ & $95 \% \mathrm{Cl}$ & $\chi^{2}$ & $P$ \\
\hline \multicolumn{6}{|l|}{ Baseline } \\
\hline \multirow[t]{4}{*}{ Cardiovascular end point } & Albuminuria & 1.17 & $1.12,1.23$ & 47.2 & $<0.001$ \\
\hline & CV medical history & 1.55 & $1.30,1.85$ & 24.2 & $<0.001$ \\
\hline & Age & 1.34 & $1.18,1.52$ & 20.1 & $<0.001$ \\
\hline & Race: Hispanic & 0.71 & $0.55,0.91$ & 7.5 & 0.006 \\
\hline \multirow[t]{4}{*}{ Heart failure } & Albuminuria & 1.26 & $1.18,1.34$ & 48.5 & $<0.001$ \\
\hline & Age & 1.46 & $1.20,1.78$ & 14.2 & $<0.001$ \\
\hline & Medical history: HF & 2.10 & $1.37,3.23$ & 11.6 & 0.001 \\
\hline & Treatment (losartan vs placebo) & 0.66 & $0.51,0.87$ & 8.7 & 0.003 \\
\hline \multicolumn{6}{|l|}{ Baseline and month- 6 change } \\
\hline \multirow[t]{5}{*}{ Cardiovascular end point } & Albuminuria & 1.19 & $1.13,1.24$ & 53.1 & $<0.001$ \\
\hline & CV medical history & 1.54 & $1.29,1.83$ & 22.9 & $<0.001$ \\
\hline & Age & 1.35 & $1.19,1.54$ & 21.8 & $<0.001$ \\
\hline & Log-change* in albuminuria & 1.32 & $1.14,1.52$ & 14.2 & $<0.001$ \\
\hline & Race: Hispanic & 0.70 & $0.54,0.90$ & 7.9 & 0.005 \\
\hline \multirow[t]{4}{*}{ Heart failure } & Albuminuria & 1.27 & $1.19,1.36$ & 53.8 & $<0.001$ \\
\hline & Age & 1.50 & $1.23,1.83$ & 16.0 & $<0.001$ \\
\hline & Log-change* in albuminuria & 1.58 & $1.25,1.98$ & 15.0 & $<0.001$ \\
\hline & Medical history: HF & 2.08 & $1.36,3.19$ & 11.3 & 0.001 \\
\hline
\end{tabular}

CV indicates cardiovascular; HF, heart failure.

${ }^{*} \mathrm{~V}=\mathrm{Log}(\mathrm{V}$ at month $6 / \mathrm{N}$ at baseline).

level at $P<0.01$ for removing a covariate from the model. The strength of a risk factor as an independent predictor for each end point was determined by its magnitude of significance using $\chi^{2}$ statistics in the multivariate analysis.

The association between albuminuria and the cardiovascular and heart failure end points was estimated using the Kaplan-Meier procedure, with baseline albuminuria stratified into 3 subgroups: $<1.5 \mathrm{~g} / \mathrm{g}, \geq 1.5<3.0 \mathrm{~g} / \mathrm{g}$, and $\geq 3.0 \mathrm{~g} / \mathrm{g}$. To estimate a risk increase between subgroups, a multivariate Cox regression model was performed with indicators of baseline albuminuria subgroups as a factor. For the adjusted analyses, the model includes other baseline covariates described above, with the exception of albuminuria and treatment group.

To estimate the effect of albuminuria change on the cardiovascular and heart failure end points, 3 groups of month- 6 albuminuria reduction were generated: $<0 \%, \geq 0<30 \%$, and $\geq 30 \%$. For the adjusted analyses, the Cox model includes indicators of 3 subgroups for reduction of albuminuria, baseline covariates previously mentioned with exception of treatment group, and the month- 6 and log-changes from baseline previously mentioned (excluding albuminuria).

To include all randomized patients into the analyses, imputed data were used in the analysis, using linear regression models with complete baseline covariates. All statistical analyses were performed using SAS version 8.

\section{Results}

\section{Baseline Albuminuria as Predictor of Cardiovascular Outcome}

Table 1 shows baseline characteristics for all patients. Mean urine albumin was $1.8 \mathrm{~g} / \mathrm{g}$ creatinine. The multivariate analysis (Table 2, top), shows that albuminuria is the strongest independent predictor of both the cardiovascular end point and heart failure.
Figure 1 (top) shows the Kaplan-Meier curves for the cardiovascular and heart failure end points for albuminuria subgroups. Both the high $(\geq 3.0 \mathrm{~g} / \mathrm{g})$ and middle $(\geq 1.5<3.0$ $\mathrm{g} / \mathrm{g}$ ) groups show significantly more cardiovascular events. Table 3 (top) summarizes the unadjusted and adjusted hazard ratios for the cardiovascular and heart failure end points. The risk for the cardiovascular end point was 1.92 -fold (95\% CI, 1.54 to 2.38 ) higher, and heart failure was 2.70 -fold (95\% CI, 1.94 to 3.75 ) higher in the high-albuminuria group compared with the low-albuminuria $(<1.5 \mathrm{~g} / \mathrm{g})$ group. To confirm that the relationship between risk for the cardiovascular end point and level of albuminuria was not driven by heart failure, we excluded the heart failure component from the cardiovascular end point. Table 3 (top) shows that the risk for the non-heart failure cardiovascular end point was similar to that of the cardiovascular end point at different levels of albuminuria.

Because of the potential for bias from arbitrary albuminuria categories, we also calculated hazard ratios for finer categories of albuminuria. Controlling for baseline risk markers, we found an almost linear positive relationship between degree of baseline albuminuria and risk for the cardiovascular end point or heart failure (Figure 1, bottom). An increase of $1 \mathrm{~g} / \mathrm{g}$ albuminuria was associated with an increased risk of $17 \%(95 \% \mathrm{CI}, 12 \%$ to $23 \%)$ for the cardiovascular end point and $26 \%$ (95\% CI, $18 \%$ to $34 \%$ ) for heart failure.

\section{Albuminuria Reduction as Predictor of Cardiovascular Outcome}

In the multivariate model (Table 2 , bottom), when adding month-6 and log-changes from baseline for the different risk 

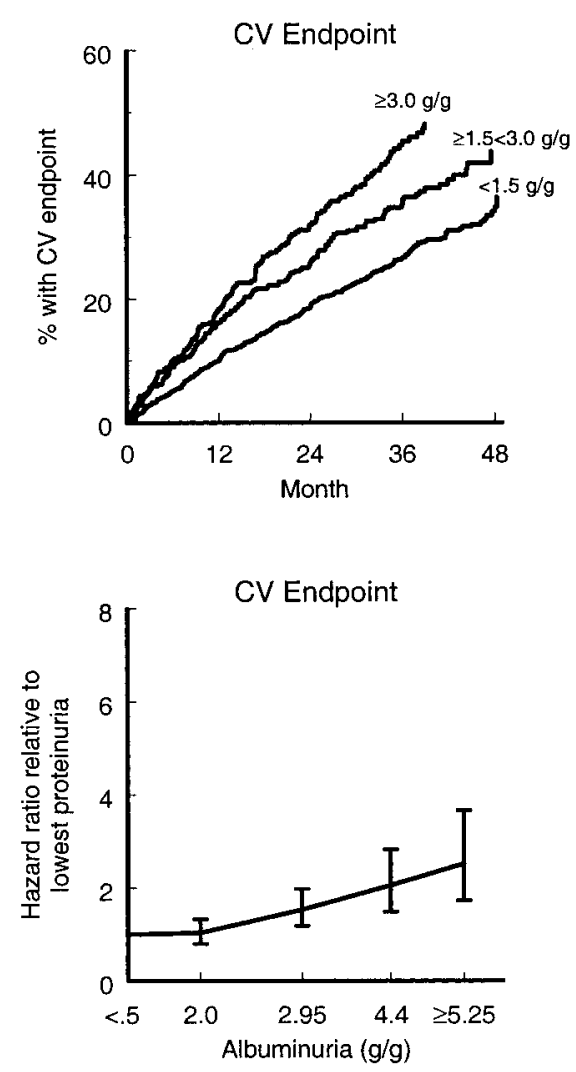
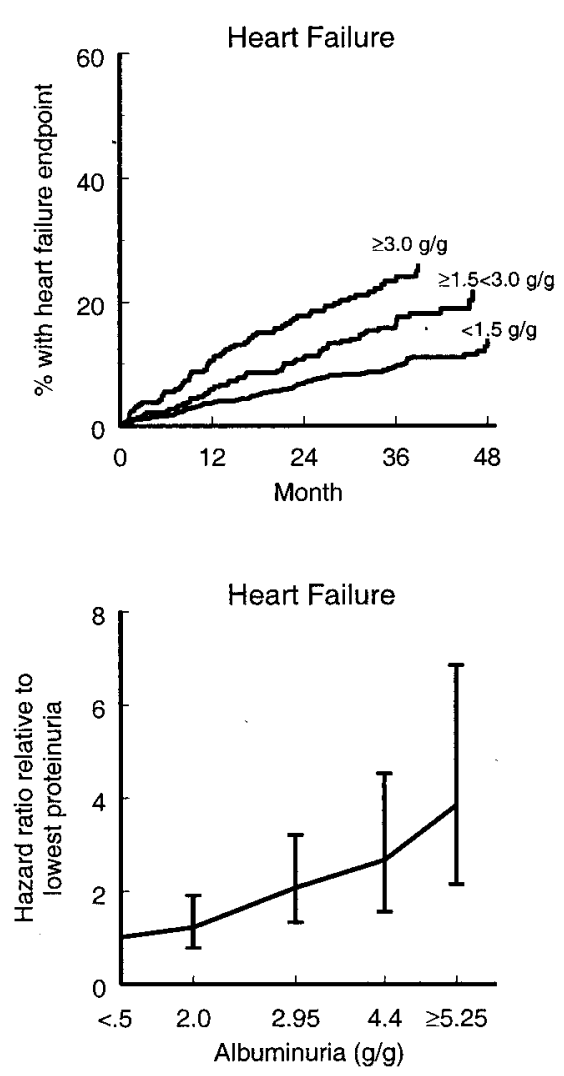

Figure 1. Top, Kaplan-Meier curves for cardiovascular and heart failure end points stratified by baseline albuminuria: $<1.5 \mathrm{~g}$ albumin $/ \mathrm{g}$ creatinine $(\mathrm{n}=859)$, $\geq 1.5<3.0 \mathrm{~g} / \mathrm{g}(\mathrm{n}=365)$, and $\geq 3.0 \mathrm{~g} / \mathrm{g}$ $(n=289)$. Bottom, Hazard ratio for cardiovascular and heart failure end points as functions of baseline albuminuria. Relation is corrected for baseline risk markers. parameters, again, baseline albuminuria is the strongest independent predictor of the cardiovascular end point or heart failure. In addition, the albuminuria reduction (log-change) is shown to be a strong predictor of cardiovascular outcome.

In the whole study population, albuminuria was lowered by $14 \%$ (95\% CI, $11 \%$ to $17 \%$ ) in the first 6 months compared with baseline. In the placebo group, it did not change significantly $(+4 \%$ [95\% CI, +8 to $-1 \%$, but it decreased by $28 \%$ (95\% CI, $25 \%$ to $36 \%$ ) in the losartan group. Because 6-month albuminuria reduction was highly variable, we subdivided the total population into 3 subgroups according to their month-6 antiproteinuric response $(<0 \%, \geq 0<30 \%$, $\geq 30 \%$ ). The cardiovascular and heart failure end points occurred more frequently in the groups that had little $(\geq 0<30 \%)$ to no $(<0 \%)$ suppression of albuminuria (Figure 2 , top). In contrast, the group that had the greatest reduction in albuminuria ( $\geq 30 \%$ ) showed a significant reduction in risk for cardiac events. The unadjusted and adjusted hazard ratios for the cardiovascular and heart failure end points are summarized in Table 3 (bottom). The risk associated with the non-heart failure cardiovascular end point was similar to that of the cardiovascular and heart failure end points.

Hazard ratios were calculated for finer categories of albuminuria reduction. Controlling for risk markers at baseline and month 6, we found an almost linear positive relationship between the degree of albuminuria reduction and risk for the cardiovascular end point or heart failure (Figure 2, bottom). Every $50 \%$ reduction in albuminuria reduces the risk for the cardiovascular end point by $18 \%$ (95\% CI, $9 \%$ to $25 \%$ ) and the heart failure end point by $27 \%$ (95\% CI, $14 \%$ to $38 \%$ ).

\section{Baseline Albuminuria and the Relation Between Cardiovascular and Renal Risk}

Figure 3 (left) shows that $28 \%$ of patients with albuminuria $<1.5 \mathrm{~g} / \mathrm{g}$ had a renal event, whereas more than $85 \%$ of patients with high albuminuria $(\geq 3.0 \mathrm{~g} / \mathrm{g}$ ) had a renal event. The level of albuminuria was associated with an increase in cardiovascular events ( $29 \%$ versus $44 \%$, respectively).

Cardiovascular and renal events could have occurred in the same patient. To identify whether the relation between albuminuria and cardiovascular events is driven by renal events, we analyzed event rates in those patients who encountered only a cardiovascular event or only a renal event, stratified by baseline albuminuria. Albuminuria is clearly associated with renal events in those subjects who did not have a cardiovascular event, whereas albuminuria shows no association with cardiovascular events in those patients who did not have a renal event (Figure 3). To exclude the phenomenon that increased cardiovascular risk is driven by ESRD, the analysis was repeated with those patients who had renal events and cardiovascular events before ESRD. Figure 3 (far right) illustrates that a pre-ESRD cardiovascular event and a renal event in the same patient are driven by albuminuria. Overall, these results indicate that an increased level of baseline albuminuria is associated with increased risk for a cardiovascular event only in those patients who also had a renal event, regardless of whether the cardiovascular event occurred before or after ESRD.

\section{Discussion}

Our results show that albuminuria is the strongest risk marker for cardiovascular events in type 2 diabetic subjects with 
TABLE 3. Risk for Cardiovascular End Points Stratified by Baseline Albuminuria and Month-6 Change in Albuminuria

\begin{tabular}{|c|c|c|c|c|c|}
\hline & \multirow[b]{2}{*}{$\mathrm{n}$} & \multicolumn{2}{|c|}{ Unadjusted } & \multicolumn{2}{|c|}{ Adjusted } \\
\hline & & $\mathrm{HR}(95 \% \mathrm{Cl})$ & $P$ & $\mathrm{HR}(95 \% \mathrm{Cl})$ & $P$ \\
\hline \multicolumn{6}{|l|}{ Baseline albuminuria, $\mathrm{g} / \mathrm{g}$} \\
\hline \multicolumn{6}{|l|}{ Cardiovascular end point } \\
\hline$\geq 1.5<3.0$ vs $<1.5$ & 365 & $1.40(1.14,1.72)$ & 0.002 & $1.46(1.19,1.80)$ & $<0.001$ \\
\hline$\geq 3.0$ vs $\geq 1.5<3.0$ & 289 & $1.26(0.99,1.61)$ & 0.059 & $1.31(1.03,1.67)$ & 0.029 \\
\hline$\geq 3.0$ vs $<1.5$ & 289 & $1.77(1.43,2.19)$ & $<0.001$ & $1.92(1.54,2.38)$ & $<0.001$ \\
\hline \multicolumn{6}{|l|}{ Heart failure end point } \\
\hline$\geq 1.5<3.0$ vs $<1.5$ & 365 & $1.74(1.26,2.41)$ & 0.001 & $1.82(1.31,2.51)$ & $<0.001$ \\
\hline$\geq 3.0$ vs $\geq 1.5<3.0$ & 289 & $1.45(1.02,2.06)$ & 0.037 & $1.48(1.04,2.11)$ & 0.028 \\
\hline$\geq 3.0$ vs $<1.5$ & 289 & $2.53(1.83,3.49)$ & $<0.001$ & $2.70(1.94,3.75)$ & $<0.001$ \\
\hline \multicolumn{6}{|c|}{ Non-heart failure cardiovascular end point } \\
\hline$\geq 1.5<3.0$ vs $<1.5$ & 365 & $1.33(1.05,1.68)$ & 0.016 & $1.38(1.10,1.75)$ & 0.006 \\
\hline$\geq 3.0$ vs $\geq 1.5<3.0$ & 289 & $1.10(0.83,1.45)$ & 0.520 & $1.13(0.85,1.49)$ & 0.404 \\
\hline$\geq 3.0$ vs $<1.5$ & 289 & $1.46(1.14,1.86)$ & 0.003 & $1.56(1.22,2.00)$ & 0.000 \\
\hline \multicolumn{6}{|l|}{ Albuminuria month- 6 change } \\
\hline \multicolumn{6}{|l|}{ Cardiovascular end point } \\
\hline$\geq 0<30 \%$ vs $<0 \%$ & 419 & $1.18(0.96,1.44)$ & 0.111 & $1.03(0.84,1.26)$ & 0.800 \\
\hline$\geq 30 \%$ vs $<0 \%$ & 458 & $0.71(0.57,0.88)$ & 0.002 & $0.66(0.53,0.83)$ & 0.000 \\
\hline$\geq 0<30 \%$ vs $\geq 30 \%$ & 458 & $0.60(0.48,0.76)$ & $<0.001$ & $0.65(0.51,0.82)$ & 0.000 \\
\hline \multicolumn{6}{|l|}{ Heart failure end point } \\
\hline$\geq 0<30 \%$ vs $<0 \%$ & 406 & $1.20(0.88,1.62)$ & 0.243 & $0.96(0.71,1.32)$ & 0.819 \\
\hline$\geq 30 \%$ vs $<0 \%$ & 475 & $0.58(0.41,0.83)$ & 0.003 & $0.51(0.36,0.73)$ & 0.000 \\
\hline$\geq 0<30 \%$ vs $\geq 30 \%$ & 475 & $0.48(0.33,0.70)$ & $<0.001$ & $0.53(0.36,0.77)$ & 0.001 \\
\hline \multicolumn{6}{|c|}{ Non-heart failure cardiovascular end point } \\
\hline$\geq 0<30 \%$ vs $<0 \%$ & 419 & $1.14(0.91,1.44)$ & 0.254 & $1.00(0.79,1.27)$ & 0.974 \\
\hline$\geq 30 \%$ vs $<0 \%$ & 458 & $0.80(0.63,1.02)$ & 0.069 & $0.77(0.60,0.98)$ & 0.031 \\
\hline$\geq 0<30 \%$ vs $\geq 30 \%$ & 458 & $0.70(0.54,0.91)$ & 0.007 & $0.76(0.59,0.99)$ & 0.042 \\
\hline
\end{tabular}

HR indicates hazard ratio. Adjusted HRs are corrected for all measured risk markers.

nephropathy. We found that the relationship between albuminuria and the cardiovascular end point was not influenced by the effect of losartan on heart failure and was present predominantly in patients with renal events. Interestingly, suppression of albuminuria was the strongest predictor of long-term protection from cardiovascular events.

There is an increasing body of evidence that albuminuria is a strong risk marker for cardiovascular disease. Results from the Framingham study demonstrated that proteinuria is associated with cardiovascular risk in the general population. ${ }^{17}$ The Steno hypothesis suggests that albuminuria is an independent risk marker of diabetic microangiopathy and macroangiopathy. ${ }^{18}$ Interestingly, Samuelsson et al ${ }^{19}$ showed that proteinuria remains a strong predictor for cardiovascular morbidity despite effective blood pressure lowering by nonRAS-blocking conventional therapies. Large clinical trials have shown that subgroups with albuminuria have a greater cardiovascular risk than those without albuminuria. ${ }^{20,21}$ Our results show that albuminuria as a risk factor for cardiovascular outcomes parallels that of renal outcomes in patients with type 2 diabetes and that this risk factor is present irrespective of conventional cardiovascular risk markers.
Since the discovery that blood pressure and serum cholesterol are independent modifiable risk markers for cardiovascular events, therapeutic strategies have been tested to lower blood pressure and serum cholesterol to reduce cardiovascular risks. ${ }^{22,23}$ Different therapeutic strategies can reduce albuminuria, including a low-protein diet, ${ }^{24}$ indomethacin, ${ }^{25}$ and antihypertensive agents such as ACEIs ${ }^{26}$ and AIIAs. ${ }^{27}$ It is of interest to determine whether these or other interventions for the reduction of albuminuria also afford cardiac protection. The presented data are the first, to the best of our knowledge, to show that lowering albuminuria is in fact related to decreased risk for cardiovascular events.

The mechanism for the relationship between albuminuria and cardiovascular risk or between the albuminuria reduction and cardiovascular protection remains unclear. Nevertheless, our results are potentially clinically important, because albuminuria is relatively easy to measure and quantify and is relatively inexpensive compared with the other strategies for measuring risks of cardiac disease and monitoring success of cardioprotective therapy effectiveness. At present, there is no parameter for monitoring the short-term therapeutic efficacy and adjusting the dose of cardioprotective therapy with RAS 

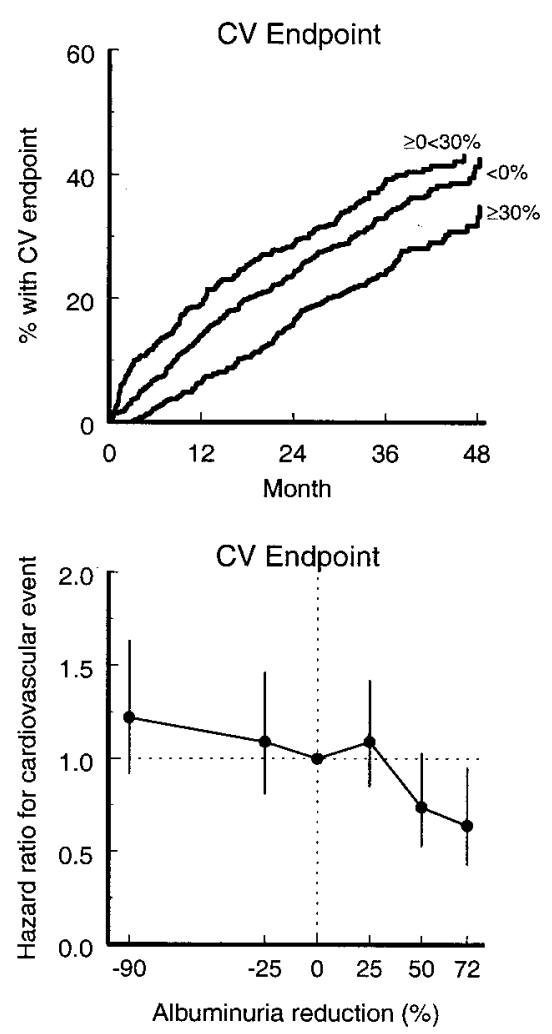
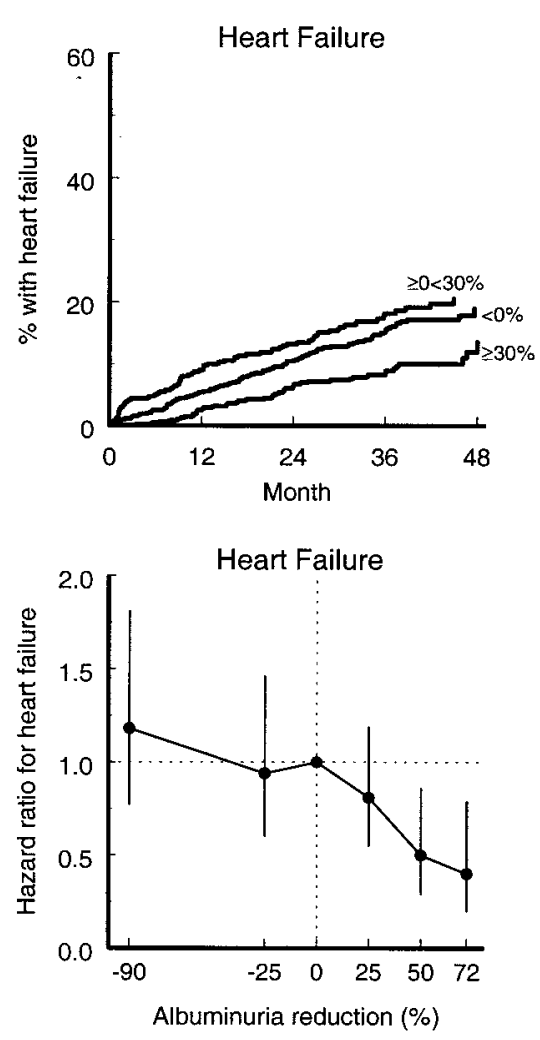

Figure 2. Top, Kaplan-Meier curves for cardiovascular and heart failure end points stratified by month- 6 change in albuminuria: $<0 \%(n=631), \geq 0<30 \%(n=393)$, and $\geq 30 \%$ ( $n=489)$. Bottom, Hazard ratio for cardiovascular and heart failure end points as functions of percent change in month- 6 albuminuria. Relation is corrected for risk markers at baseline and month- 6 change and log-change. intervention, but if prospectively validated, albuminuria could potentially serve these purposes.

Assessing the effect of treatment on reduction of albuminuria was a secondary end point in this trial; however, evaluating the relationship between albuminuria and the cardiovascular or heart failure end points was not prespecified. The arbitrary choice of cut points for baseline and reduction in albuminuria could have influenced the results; however, the outcome of the analyses was similar for the cardiovascular end points using tertile and quintile cut points (data not shown). Although these analyses indicate a strong

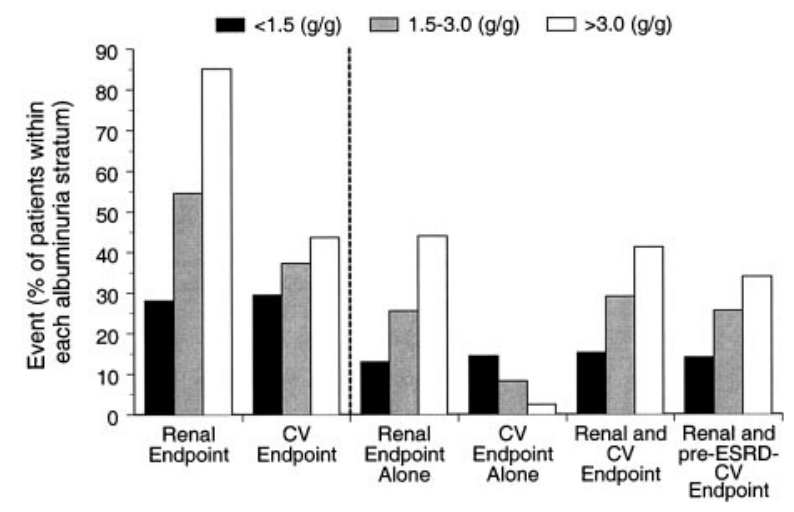

Figure 3. Relation between cardiovascular and renal events in different albuminuria subgroups. All patients stratified by baseline albuminuria: $<1.5 \mathrm{~g} / \mathrm{g}(\mathrm{n}=859), 1.5$ to $3.0 \mathrm{~g} / \mathrm{g}(\mathrm{n}=365)$, and $\geq 3.0 \mathrm{~g} / \mathrm{g}(\mathrm{n}=289)$. Left, patients with renal events or cardiovascular (CV) events. Right, Patients with renal events alone, cardiovascular events alone, renal and cardiovascular events in same patient, and renal and pre-ESRD CV events in same patient. association between albuminuria and cardiovascular outcome, this association does not imply causality. Because the effect of treatment on reduction of albuminuria per se was not the primary aim of the RENAAL study, the findings presented in this article cannot be considered definitive evidence that treatment with the goal of reducing albuminuria will reduce the risk for cardiovascular events.

These results extend the concept that suppressing albuminuria should be evaluated further as a goal of therapy to achieve optimal cardiovascular protection in the individual patient with type 2 diabetes. Because the dose-response curves for blood pressure and albuminuria appear to be different, ${ }^{28,29}$ it is possible that therapy aimed at reducing albuminuria could result in additional benefit beyond that achieved with blood pressure lowering alone. These results emphasize the need for new studies in which the primary target of therapy is reduction of albuminuria in other diseases affecting the kidney.

\section{Disclosure}

Dr de Zeeuw has served as a consultant to Merck, Bristol-Myers Squibb, Sanofi, Pfizer, Actelion, and Solvay. Dr Remuzzi has received research grants from Aventis Pharma, Abbott, and Novartis. He has been a member of speakers' bureaus sponsored by Merck, AstraZeneca, Novartis, and Aventis Pharma. Dr Parving has served as a consultant to Merck, Bristol-Meyers Squibb, Sanofi, Pfizer, and BioStratum; has received research grants from Merck, BristolMeyers Squibb, Sanofi, and AstraZeneca; and has been a member of speakers' bureaus sponsored by Merck, Bristol-Meyers Squibb, Sanofi, Pfizer, and AstraZeneca. Dr Cooper has served as consultant to Pfizer, Sanofi, and Alteon. He has received research grants from AstraZeneca, Merck, Bristol-Myers Squibb, and Servier. He has been a member of speakers' bureaus sponsored by AstraZeneca and Solvay. Dr Mitch has served as a consultant to Merck. Drs Keane, 
Zhang, Shahinfar, and Snapinn are employees of Merck \& Co, Inc, and potentially own stock and/or hold stock options in the Company.

\section{References}

1. King H, Aubert R, Herman W. Global burden of diabetes 1995-2025: prevalence, numerical estimates and projections. Diabetes Care. 1998; 21:1414-1431.

2. Amos A, McCarty D, Zimmet P. The rising global burden of diabetes and its complications: estimates and projections to the year 2010. Diabet Med. 1997;14(S 5):S1-S85.

3. Morrish NJ, Wang SL, Stevens LK, et al. Mortality and causes of death in the WHO Multinational Study of Vascular Disease in Diabetes. Diabetologia. 2001;44(suppl 2):S14-S21.

4. Parving H-H. Diabetic nephropathy: prevention and treatment. Kidney Int. 2001;60:2041-2055.

5. Remuzzi G, Schieppati A, Ruggenenti P. Nephropathy in patients with type 2 diabetes. N Engl J Med. 2002;346:1145-1151.

6. American Diabetes Association. Management of dyslipidemia in adults with diabetes. Diabetes Care 2003;26(suppl 1):S83-S86.

7. Stamler J, Vaccaro O, Neaton JD, et al. Diabetes, other risk factors, and 12-yr cardiovascular mortality for men screened in the Multiple Risk Factor Intervention Trial. Diabetes Care. 1993;16:434-444.

8. Tight blood pressure control and risk of macrovascular and microvascular complications in type 2 diabetes: UKPDS 38. UK Prospective Diabetes Study Group. BMJ. 1998;317:703-713.

9. Rossing P, Hougaard P, Borch-Johnsen K, et al. Predictors of mortality in insulin dependent diabetes: 10 year observational follow up study. BMJ. 1996;313:779-784.

10. Wang SL, Head J, Stevens L, et al. Excess mortality and its relation to hypertension and proteinuria in diabetic patients. The World Health Organization Multinational Study of Vascular Disease in Diabetes. Diabetes Care. 1996;19:305-312.

11. Keane WF, Brenner BM, de Zeeuw D, et al. The risk of developing end-stage renal disease in patients with type 2 diabetes and nephropathy: the RENAAL Study. Kidney Int. 2003;63:1499-1507.

12. The GISEN Group. Randomised placebo-controlled trial of effect of ramipril on decline in glomerular filtration rate and risk of terminal renal failure in proteinuric, non-diabetic nephropathy. Lancet. 1997;349: 1857-1863.

13. Apperloo AJ, De Zeeuw D, De Jong PE. Short-term antiproteinuric response to antihypertensive treatment predicts long-term GFR decline in patients with non-diabetic renal disease. Kidney Int 1994;45(suppl 45): S174-S178.

14. de Zeeuw D, Remuzzi G, Parving HH, et al. Proteinuria, a target for renoprotection in patients with type 2 diabetic nephropathy: lessons from RENAAL. Kidney Int. 2004;65:2309-2320.
15. Brenner BM, Cooper MA, de Zeeuw D, et al. Effects of losartan on renal and cardiovascular outcomes in patients with type 2 diabetes and nephropathy. N Engl J Med. 2001;345:861-869.

16. Brenner BM, Cooper ME, de Zeeuw D, et al. The Losartan Renal Protection Study: rationale, study design and baseline characteristics of RENAAL (Reduction of Endpoints in NIDDM with the Angiotensin II Antagonist Losartan). J Renin Angiotensin Aldosterone Syst. 2000;1: $328-335$.

17. Kannel WB, Stampfer MJ, Castelli WP. The prognostic significance of proteinuria: the Framingham study. Am Heart J. 1984;108:1347-1352.

18. Deckert T, Feldt-Rasmussen B, Borch-Johnsen K, et al. Albuminuria reflects widespread vascular damage: the Steno hypothesis. Diabetologia. 1989;32:219-226.

19. Samuelsson O, Wilhelmsen L, Pennert K, et al. Prognostic factors in treated hypertension. J Hypertens. 1985;3(suppl 3):S497-S500.

20. Hansson L, Zanchetti A, Carruthers SG, et al. Effects of intensive bloodpressure lowering and low-dose aspirin in patients with hypertension: principal results of the Hypertension Optimal Treatment (HOT) randomized trial. Lancet. 1998;351:1755-1762.

21. The Heart Outcomes Prevention Evaluation Study Investigators. Effects of an angiotensin-converting-enzyme inhibitor, ramipril, on cardiovascular events in high-risk patients. N Engl J Med. 2000;342:145-153.

22. The ALLHAT Officers and Coordinators for the ALLHAT Collaborative Research Group. Major outcomes in high-risk hypertensive patients randomized to angiotensin-converting enzyme inhibitor or calcium channel blocker vs diuretic. The Antihypertensive and Lipid-Lowering Treatment to Prevent Heart Attack Trial (ALLHAT). JAMA. 2002;288:2981-2997.

23. Randomized trial of cholesterol lowering in 4444 patients with coronary heart disease: the Scandinavian Simvastatin Survival Study (4S). Lancet. 1994;344:1383-1389.

24. El Nahas AM, Masters-Thomas A, Brady SA, et al. Selective effect of low protein diets in chronic renal diseases. BMJ. 1984;289:1337-1341.

25. Vriesendorp R, Donker AJM, de Zeeuw D, et al. Effects of nonsteroidal anti-inflammatory drugs on proteinuria. Am J Med. 1986;81(suppl 2B): 84-94.

26. Gansevoort RT, Sluiter WJ, Hemmelder MH, et al. Antiproteinuric effect of blood-pressure-lowering agents: a meta-analysis of comparative trials. Nephrol Dial Transplant. 1995;10:1963-1974.

27. Gansevoort RT, de Zeeuw D, de Jong PE. Is the antiproteinuric effect of ACE inhibition mediated by interference in the renin-angiotensin system? Kidney Int. 1994;45:861-867.

28. Laverman GD, Henning RH, De Jong PE, et al. Optimal antiproteinuric dose of losartan in non-diabetic patients with nephrotic range proteinuria. Am J Kidney Dis. 2001;38:1381-1384.

29. Andersen S, Rossing P, Juhl TR, et al. Optimal dose of losartan for renoprotection in diabetic nephropathy. Nephrol Dial Transpl. 2002;17: 1413-1418. 